\title{
The Structure of the (100) Surface of Ag-In-Gd 1/1 Approximant
}

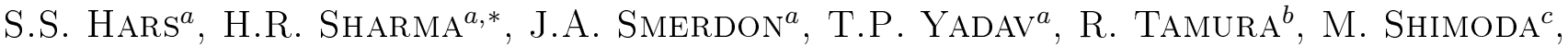 \\ AND R. MCGRATH ${ }^{a}$ \\ ${ }^{a}$ Surface Science Research Centre and Department of Physics, The University of Liverpool, Liverpool L69 3BX, UK \\ ${ }^{b}$ Department of Materials Science and Technology, Tokyo University of Science, Noda, Japan \\ ${ }^{c}$ National Institute for Materials Science, Sengen, Tsukuba, Ibaraki 305-0047, Japan
}

\begin{abstract}
Scanning tunneling microscopy is employed to characterise the structure and morphology of the (100) surface of the Ag-In-Gd 1/1 approximant. The surface prepared by the usual method of sputter-annealing produces stepterrace structure. Observed step heights are consistent with the lattice constant of the bulk. Scanning tunneling microscopy on terraces reveals cluster-like protrusions arranged with a square unit cell as expected from the bulk. It has not been possible to assign the terraces to the specific bulk planes because of lack of atomic resolution on terraces.
\end{abstract}

DOI: $10.12693 /$ APhysPolA.126.479

PACS: 61.44.Br, 68.35.Bs, 68.37.Ef, 71.23.Ft

\section{Introduction}

Quasicrystals are metallic alloys which show longrange order but without periodicity or translational symmetry. They can possess classically forbidden rotational symmetries [1]. Crystalline approximants are metallic compounds whose chemical composition and atomic structure are close to their parent quasicrystals $[2-5]$. They possess large periodic unit cells [6]. Crystalline approximants can play an important role in understanding the structure of quasicrystals because their structure can be determined using conventional structure analysis techniques $[7,8]$.

The binary icosahedral Cd-Yb quasicrystal $[9,10]$ has attracted great attention because of the lack of chemical disorder in the structure and large number of related approximants that have been found. The study of their surfaces would open up a new area of investigation in the surface science of quasicrystals. However samples containing $\mathrm{Cd}$ cannot be prepared in ultra-high vacuum (UHV) because of high vapour pressure of $\mathrm{Cd}$ [11]. This problem has been solved by replacing $\mathrm{Cd}$ with equal amounts of $\mathrm{Ag}$ and In yielding new ternary quasicrystals and approximants such as the $i$ - $\mathrm{Ag}-\mathrm{In}-\mathrm{Yb}$ and the $\mathrm{Ag}$ $\mathrm{In}-\mathrm{Yb} 1 / 1$ approximant [12]. Replacing $\mathrm{Yb}$ with other rare earth elements generates further crystalline approximants such as Ag-In-Gd, Ag-In-Tb [13] and Ag-In-Eu $1 / 1[7]$.

The crystal structure of $1 / 1$ approximants is the bodycentered-cubic packing of rhombic triacontahedral (RTH) clusters, which is the basic building block of the Ag-In-Gd $1 / 1$ approximant. This RTH cluster has five successive

*corresponding author; e-mail: H.R.Sharma@liverpool.ac.uk atomic shells. The inner shell is a tetrahedron with $4 \mathrm{Cd}$ atoms. The second shell is a dodecahedron which has 20 $\mathrm{Cd}$ atoms, the third shell is an icosahedron with $12 \mathrm{Yb}$ atoms, and the fourth shell is an icosidodecahedron with $30 \mathrm{Cd}$ atoms. Finally, the outer-most shell is a rhombic triacontahedron with $92 \mathrm{Cd}$ atoms [5, 9].

In this study, the (100) surface of the Ag-In-Gd 1/1 approximant has been characterised using scanning tunneling microscopy (STM). The goal is to understand the clean (100) surface of Ag-In-Gd 1/1 approximant and to prepare it for use as a template to grow new metallic and molecular thin films. This will help in understanding the factors affecting epitaxy on these approximants and provide a useful comparison to growth on the $i$-Ag-In- $\mathrm{Yb}$ quasicrystal surfaces.

\section{Experimental details}

The sample was grown using the self-flux method. The sample develops facets along (100) surface during the growth. The sample was hand polished along the (100) surface using $6 \mu \mathrm{m}, 1 \mu \mathrm{m}$ and $0.25 \mu \mathrm{m}$ diameter diamond pastes successively. The sample was not cut along (100) because of its tiny size. Following this, the surface was cleaned in ultra-high vacuum (UHV) using sputtering at room temperature by $\mathrm{Ar}^{+}(2.5-3 \mathrm{keV})$ for 30 minutes followed by annealing $\left(450^{\circ} \mathrm{C}\right)$ for 4 hours under a base pressure of $2 \times 10^{-10}$ mbar. An infra-red pyrometer with emissivity of 0.35 was used to monitor the temperature. This preparation produced a step/terrace morphology and showed facets on the surface. The surface orientation was checked by the Laue backscattering after the UHV experiments. It was found that the surface is tilted by $10^{\circ}$ from the [001] and $4^{\circ}$ from [010].

We consider that the surface composition after annealing is similar to the bulk based on the following observations on the isostructural Ag-In-Yb system. XPS stud- 
ies from the $i$-Ag-In-Yb quasicrystal, which has a composition close to the $\mathrm{Ag}-\mathrm{In}-\mathrm{Yb} 1 / 1$ approximant, shows a depletion of In and $\mathrm{Yb}$ upon sputtering but the bulk chemical composition is regained at the surface after annealing at around $350{ }^{\circ} \mathrm{C}$ [14]. This surface exhibits a step-terrace structure consistent with bulk terminations after annealing at $430-440{ }^{\circ} \mathrm{C}$ [15]. Similarly, the surface composition of the (100) surface of the Ag-In-Yb 1/1 approximant also shows a surface composition similar to the bulk after annealing at around $440^{\circ} \mathrm{C}$ [16].

Scanning tunneling microscopy at room temperature was used to record images of the surfaces using an Omicron VT-STM instrument. The bulk model structure of the $\mathrm{Cd}-\mathrm{Tb} 1 / 1$ approximant, isostructural to the $\mathrm{Ag}$ In-Tb approximant, was used to compare STM images. VESTA is used to analyse the model structure.

\section{Results and discussion}

The surface develops facets along different crystallographic directions. The detailed analysis of facets will be published elsewhere. Here we focus on the structure of the (100) surface. The formation of facets may be due to misorientation of the surface. The (100) surface of isostructural $\mathrm{Ag}$-In- $\mathrm{Yb}$ with perfect orientation did not exhibit any facets.

Figure 1a shows a $60 \times 60 \mathrm{~nm}^{2}$ STM image of the (100) surface. The line profile in Fig. 1b shows that the flat terraces are separated by $7.7 \pm 0.04 \AA$ high steps. A $\mathrm{Cd}-\mathrm{Yb}$ unit cell is given in Fig. 1c for comparison. The structure can be described by flat and puckered layers. The flat layers intersect the faces and body centre of the cube, i.e., $z=0$ and $a / 2, a$ being the lattice constant. The puckered layers are at 2.46 and $5.19 \AA$. The position of the puckered layer is taken at the region where the atomic density is high. Other layers at $z>a / 2$ are mirror planes of the layers at $z<a / 2$. The distance between flat layers is $7.67 \AA$, which is close to the step height. However, the separation of other planes also have similar value (some of them are marked in 1c). Therefore, it is not possible to assign the terraces to the bulk plane based on the step height distribution alone.

High resolution STM images reveal protrusions on the terraces, arranged with a square unit cell, as shown in Fig. 2a. The distance between protrusions $(15.20 \AA)$ is obtained by measuring the peak to peak distance between adjacent maxima in line profiles. This value is in good agreement with the bcc lattice constant for the Ag-In-Gd $1 / 1$ approximant of $15.29 \AA[13]$. The fast-Fourier transform (FFT) of the STM image, not shown, is compatible with the square lattice, and also indicates that the surface is well ordered. The size of each protrusion is about $1 \mathrm{~nm}$, i.e., no atomic resolution could be obtained and thus it is not possible to compare STM and model on atomic scale.

Now we compare the surface structure of $\mathrm{Ag}-\mathrm{In}-\mathrm{Gd}$ with the same surface of the isostructural Ag-In-Yb [17] and $\mathrm{Ag}-\mathrm{In}-\mathrm{Tb} 1 / 1$ approximants [18]. A study reported
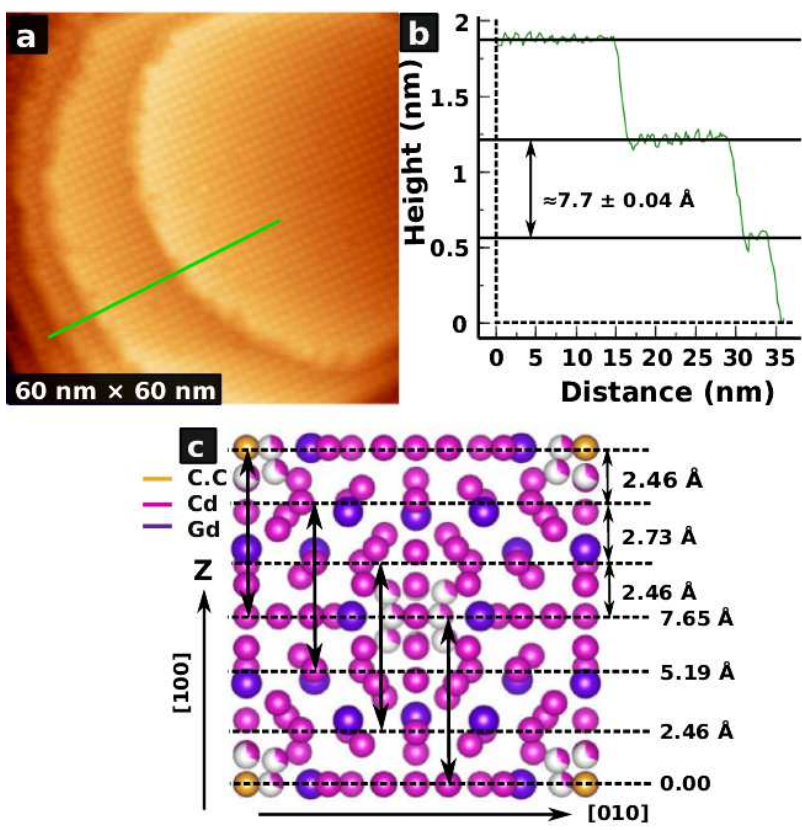

Fig. 1. (a) $60 \times 60 \mathrm{~nm}^{2} \mathrm{STM}$ image showing a terrace/step morphology on the Ag-In-Gd (100) surface; (b) a line profile across the terraces of image (a); (c) a unit cell of the Cd-Gd 1/1 approximant.

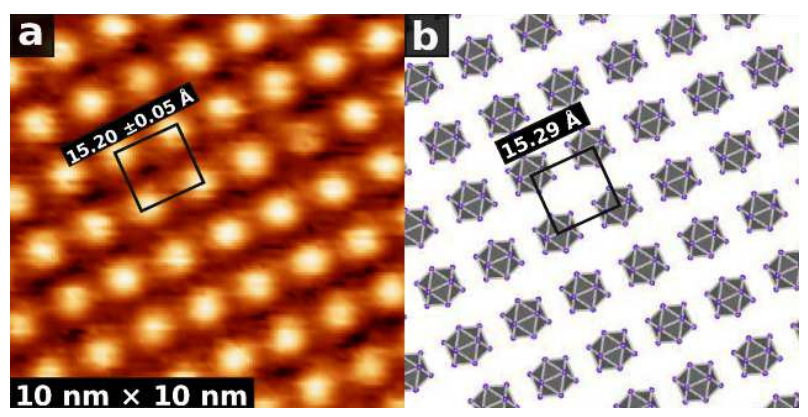

Fig. 2. (a) $10 \times 10 \mathrm{~nm}^{2}$ STM image showing clusters on the Ag-In-Gd (100) surface; (b) $\mathrm{Cd}_{6} \mathrm{~Tb} 1 / 1$ approximant bulk structure model showing square unit cell linking clusters.

by Cui et al. on the (100) surface of the Ag-In-Yb 1/1 approximant shows step heights of 2.1,3.1 and 7.3 $\AA$ [17]. The lower step heights are in agreement with the distance between consecutive layers in the unit cell. These steps are observed on the surface annealed at slightly lower temperature and for shorter time than the current study. Under the employed surface preparation, the Ag-In-Gd surface did not show such lower step heights. All three surfaces show protrusions on terraces, no atomic resolution was obtained. Based on the step height distribution and structure on the terraces, it is concluded that flat layers are more stable than puckered layers in the Ag-InYb approximant [16].

In addition to the STM images described above, we occasionally observed images, which cannot be explained 
by the model structure. Figure 3 shows such an STM image of $100 \times 100 \mathrm{~nm}^{2}$ with flat but featureless terraces separated by $2.60 \AA$ high steps. The step heights are not consistent with those expected from the separation of the bulk planes. From the model, steps of two different heights are expected to be observed (refer to in Fig. 1c). The Fourier transform of the terraces reveals no ordered structure. It is not clear whether these STM images are from a surface of a different crystalline phase or surface of the same phase but with different surface orientation.

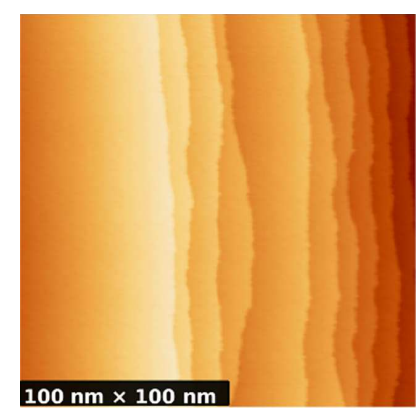

Fig. 3. $100 \times 100 \mathrm{~nm}^{2}$ STM images showing a different terrace/step morphology than that predominant morphology observed on the (100) surface of the 1/1 Ag-InGd approximant.

\section{Conclusions}

Scanning tunneling microscopy (STM) has been used to characterise the surface structure of the Ag-In-Gd 1/1 approximant. The surface, which is slightly off from (100), produces facets along various crystallographic directions. The structure of the (100) facets is analysed. The observed step heights are consistent with the lattice constant of the bulk. Terraces reveal protrusions arranged with a square unit cell as expected from the bulk symmetry. However, no atomic resolution was obtained. The step heights and protrusions are similar to those observed in the isostructural Ag-In-Yb $1 / 1$ approximant, where the surface terminates at flat high atomic density planes.

\section{References}

[1] D. Shechtman, I. Blech, D. Gratias, J.W. Cahn, Phys. Rev. Lett. 53, 1951 (1984).

[2] P.A. Thiel, Prog. Surf. Sci. 75, 69 (2004).

[3] P.A. Thiel, Ann. Rev. Phys. Chem. 59, 129 (2008).

[4] A.I. Goldman, R.F. Kelton, Rev. Mod. Phys. 65, 213 (1993).

[5] A.P. Tsai, C.P. Gomez, Handbook of metal physics, vol. 3, Elsevier, Amsterdam 2008.

[6] H.R. Sharma, M. Shimoda, A. P. Tsai, Adv. Phys. 56, 403 (2007).

[7] C. Cui, A.P. Tsai, Philos. Mag. 91, 2443 (2011).

[8] P.A. Thiel, J. Alloys Comp. 536, 91 (2012).

[9] A.P. Tsai, J.Q. Guo, E. Abe, H. Takakura, T.J. Sato, Nature 408, 537 (2000).

[10] J. Q. Guo, E. Abe, A. P. Tsai, Phys. Rev. B 62, 14605 (2000).

[11] S. Ohhashi, J. Hasegawa, S. Takeuchi, A.P. Tsai, Philos. Mag. 87, 3089 (2007).

[12] J.Q. Guo, A.P. Tsai, Philos. Mag. Lett. 82, 349 (2002).

[13] R. Tamura, to be published.

[14] H.R. Sharma, M. Shimoda, S. Ohhashi, A.P. Tsai, Philos. Mag. 87, 2989 (2007).

[15] H.R. Sharma, M. Shimoda, K. Sagisaka, H. Takakura, J.A. Smerdon, P.J. Nugent, R. McGrath, D. Fujita, S. Ohhashi, A.P. Tsai, Phys. Rev. B 80, 121401 (2009).

[16] K. Nozawa, C. Cui, M. Shimoda, P.J. Nugent, H.R. Sharma, Y. Ishii, to be published.

[17] C. Cui, H.R. Sharma, P.J. Nugent, M. Shimoda, A.P. Tsai, accepted for publication.

[18] S.S. Hars, J.A. Smerdon, T.P. Yadav, R. Tamura, R. McGrath, H. R. Sharma, to be published. 